\title{
Global Correlation Clustering Based on the Hough Transform
}

\author{
Elke Achtert, Christian Böhm, Jörn David, Peer Kröger, Arthur Zimek* \\ Department Institute for Informatics, Ludwig-Maximilians Universität München \\ Received 4 April 2008; revised 30 May 2008; accepted 8 June 2008 \\ DOI:10.1002/sam.10012 \\ Published online 3 November 2008 in Wiley InterScience (www.interscience.wiley.com).

\begin{abstract}
In this article, we propose an efficient and effective method for finding arbitrarily oriented subspace clusters by mapping the data space to a parameter space defining the set of possible arbitrarily oriented subspaces. The objective of a clustering algorithm based on this principle is to find those among all the possible subspaces that accommodate many database objects. In contrast to existing approaches, our method can find subspace clusters of different dimensionality even if they are sparse or are intersected by other clusters within a noisy environment. A broad experimental evaluation demonstrates the robustness and effectiveness of our method. (c) 2008 Wiley Periodicals, Inc. Statistical Analysis and Data Mining 1: 111-127, 2008
\end{abstract}

Keywords: high dimensional data; subspace clustering; correlation clustering

\section{INTRODUCTION}

Subspace clustering is a data-mining task, which has attracted considerable attention during the last years. There are two main reasons for this popularity. Firstly, conventional (full space) clustering algorithms often fail to find useful clusters when applied to datasets of higher dimensionality, because typically, many of the attributes are noisy, some attributes may exhibit correlations among another, and only few of the attributes really contribute to the cluster structure. Secondly, the knowledge gained from a subspace-clustering algorithm is much richer than that of a conventional clustering algorithm. It can be used for interpretation, data compression, similarity search, etc. as we will discuss in the next paragraph.

We can distinguish between subspace-clustering algorithms for axis-parallel subspaces [1-5] and those for subspaces which are arbitrarily oriented (called oriented clustering, generalized subspace clustering, or correlation clustering, e.g. [6-8]). In both cases, the data objects which are grouped into a common subspace cluster, are very dense (i.e. the variance is small) when projected onto the hyperplane which is perpendicular to the subspace of the cluster (called the perpendicular space plane). The objects may form a completely arbitrary shape with a high variance when projected onto the hyperplane of the subspace in which the cluster resides (called the cluster subspace plane). This means, that the objects of the subspace cluster

Correspondence to: Arthur Zimek (zimek@dbs.ifi.lmu.de) are all close to the cluster subspace plane. The knowledge that all data objects of a cluster are close to the cluster subspace plane is valuable for many applications: If the plane is axis-parallel, this means that the values of some of the attributes are more or less constant for all cluster members. The whole group is characterized by this constant attribute value, an item of information which can definitely be important for the interpretation of the cluster. This property may also be used to perform a dedicated dimensionality reduction for the objects of the cluster and may be useful for data compression (because only the higher-variance attributes must be stored at high precision individually for each cluster member) and similarity search (because only the high-variance attributes need to be individually considered for the search, and an index needs only to be constructed for the high-variance attributes).

If the cluster subspace plane is arbitrarily oriented, the knowledge is even more valuable. In this case, we know that the attributes which define the cluster subspace plane, have a complex dependency among each other. This dependency defines a rule, which again characterizes the cluster and which is potentially useful for cluster interpretation. Similar to the case of axis-parallel clusters, this dependency rule may also be used for dimensionality reduction, data compression, similarity search, and indexing. Consider, for example, Fig. 1 which contains two general subspace clusters in a very noisy environment. For each of the subspace clusters, we know that the $x$ and $y$ coordinates are approximately linearly dependent from each other $\left(y \approx m_{i} \cdot x+t_{i}\right)$, and, therefore, only one of them needs 


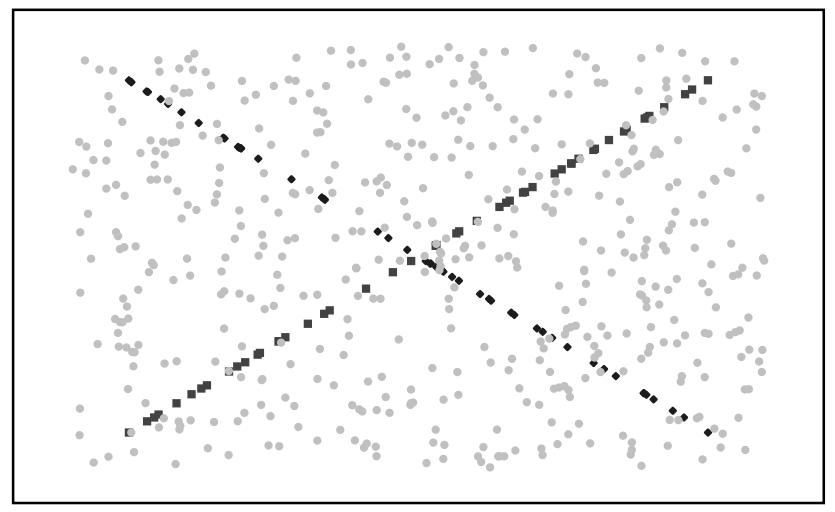

Fig. 1 Dataset with two nondense general subspace clusters in a noisy environment.

to be stored at full precision, indexed, etc. Furthermore, the knowledge of the degree of dependency, as well as the slope and intercept may be important for the interpretation of the cluster in the context of the application.

One well-known effect of the "curse of dimensionality' is the correlation among attributes in high-dimensional data. While full-dimensional clustering approaches are easily misled by these correlations, generalized subspaceclustering approaches, hence also called correlation clustering, make use of this effect to identify clusters in subspaces of arbitrary dimensionality. However, finding axis-parallel or generally oriented subspace clusters is not a trivial task. The number of possible axis parallel subspaces is exponential in the number of dimensions, and the number of general subspaces is even infinite. Therefore, a complete enumeration of all possible subspaces to be checked for clusters is not feasible. Consequently, all previous solutions rely on specific assumptions and heuristics and try to find promising subspaces during the clustering process, for instance, in an iterative optimization. We will see that this previous approach of learning suitable subspaces works well if (but only if) subspace clusters are locally well separated and no outlier objects (belonging to any cluster) exist. In the presence of outliers in the local neighborhood of cluster points or cluster representatives in the entire feature space, most previous subspace-clustering algorithms fail to detect subspace clusters, because the algorithms try to find suitable subspaces for each cluster from the local neighborhood of cluster points or cluster representatives in the entire feature space. This fundamental assumption that all existing approaches to correlation clustering are based upon is called the 'locality assumption'. Outliers in the neighborhoods, that do not belong to the corresponding cluster prevent the algorithms from finding suitable subspaces, and the absence of a precise subspace prevents the algorithm from effectively filtering out the outliers.
In high-dimensional spaces, however, where distances cannot be used to differentiate between near and far points, the concept of local neighborhoods is meaningless $[9,10,11]$. Consequently, the neighborhoods of cluster points or cluster representatives will contain a large number of outliers that do not belong to the corresponding cluster. However, those problems arise even if the number of outliers is very small (e.g. 5-10 outliers in the complete dataset-see e.g. [12]). Thus, an environment of heavy noise such as that of Fig. 1 is completely out of the scope of previous subspace-clustering methods even in lower-dimensional data spaces, as we will discuss more deeply in Section 2 for locally optimizing approaches such as ORCLUS [6], and for density-based approaches such as $4 \mathrm{C}$ [7].

To escape from this circular dependency of subspace finding and outlier filtering, we propose in this article to reconsider the problem of finding generally oriented subspace clusters from a new perspective. The main idea is to transform every object into a new space, the space of all possible subspaces in which this object is contained. Since the number of all such subspaces is infinite, we neither enumerate these subspaces nor represent each object by an infinite (or very high) number of transformed objects. Instead, we consider a continuum of many possible subspaces represented by a small number of parameters. This continuum is split up on demand during the clustering process to set limits to the allowed cluster subspace planes and finally identify subspace clusters. We will describe this method in detail in Section 3, and then experimentally evaluate our method in Section 4. Section 5 concludes our article.

\section{RELATED WORK}

Existing approaches for subspace clustering rely on certain heuristics that use specific assumptions to shrink the search space and thus reduce the runtime complexity. However, if these assumptions are not true for a given dataset, the effectiveness of these methods will either deteriorate, or the method will even fail to detect any suitable patterns, or the methods exhibit an exponential runtime, and a good performance in terms of effectiveness is paid for in terms of efficiency.

Many subspace-clustering algorithms (e.g. [1,2,3,4,5, $13,14]$ ) assume that the subspace clusters are axis-parallel. Otherwise, they will not find any pattern. Pattern-based subspace-clustering algorithms (e.g. $[15,16,17,18,19])$ are limited to finding only clusters that represent pairwise positive correlations in the dataset. In contrast, arbitrarily oriented hyperplanes (subspace clusters) may also represent more complex or negative correlations. 
In this article, we focus on the generalized problem of finding arbitrarily oriented subspace clusters. All existing algorithms for this problem assume that the cluster structure is significantly dense in the local neighborhood of the cluster centers or other points that participate in the cluster. In the context of high-dimensional data, this 'locality assumption' is rather optimistic. Theoretical considerations [10] show that concepts like 'local neighborhood' are not meaningful in high-dimensional spaces because distances can no longer be used to differentiate between points. This is a consequence of the well-known curse of dimensionality.

ORCLUS [6] is based on $k$-means and iteratively learns the similarity measure capturing the subspace containing a given cluster from the points assigned to the cluster in each iteration by applying principal component analysis (PCA) on these points. Since the algorithm starts with the Euclidean distance, the algorithm learns the subspaces from the local neighborhood of the initial cluster centers. However, if this local neighborhood contains some noise or the clustering structure is too sparse within this local neighborhood, the learning heuristic will be misled because PCA is rather sensitive to outliers. In those cases, ORCLUS will fail to detect meaningful patterns. These considerations, accordingly, apply to the method proposed in [20] which is a slight variant of ORCLUS designed for enhancing multidimensional indexing.

4C [7] integrates PCA into density-based clustering. It evaluates the Euclidean neighborhood of each point $p$ to learn the subspace characteristics in which $p$ can be clustered best. Similar to ORCLUS, 4C thus relies on the assumption that the clustering structure is dense in the entire feature space. Otherwise, $4 \mathrm{C}$ will also fail to produce meaningful results. The same holds true of some variations of 4C, like HiCO [21], COPAC [22], and ERiC [23], and also for robustified versions of these algorithms as described in [12].

The method CURLER [8] merges the clusters computed by the EM algorithm using the so-called cosharing level. The resulting clusters need not represent linear correlations. Rather, any dense pattern in the data space is found that may represent a more complex, not necessarily linear correlation. CURLER also relies on the assumption that the subspaceclustering structure is dense in the entire feature space because both the generation as well as the merging of microclusters uses local neighborhood information.

Let us note that the term 'correlation clustering' relates to a different task in the machine-learning community, where a partitioning of the data correlates as much as possible with a pairwise similarity function $f$ learned from past data (e.g. cf. [24]).

\section{ALGORITHM CASH}

Obviously, the locality assumption that the clustering structure is dense in the entire feature space, and that the Euclidean neighborhood of points in the cluster, or of cluster centers, does not contain noise is a very strict limitation for high-dimensional real-world datasets. In [10] the authors show that in high-dimensional spaces, the distance to the nearest neighbor and the distance to the farthest neighbor converge. As a consequence, distances can no longer be used to differentiate between points in highdimensional spaces and concepts like the neighborhood of points become meaningless. Usually, although many points share a common hyperplane, they are not close to each other in the original feature space. In those cases, existing approaches will fail to detect meaningful patterns because they cannot learn the correct subspaces of the clusters. In addition, as long as the correct subspaces of the clusters cannot be determined, obviously outliers and noise cannot be removed in a preprocessing step.

In this article, we propose to use the ideas of the Hough transform [25] to develop an original principle for characterizing the subspace containing a cluster.

The basic Hough transform has been introduced in the computer graphics community to address the problem of finding linear segments in pictures (especially straight lines) by [26]. Most work focuses on discretized twodimensional data. The key idea is to map each point of a two-dimensional picture (or data space $\mathcal{D}$ ) such as a pixel onto a set of points (e.g. a line) in a parameter space $\mathcal{P}$. An area of the parameter space containing many mapped points (e.g. the intersection of many lines) indicates a potential feature of interest. In general, a linear segment $s$ can be represented by its slope $m_{s}$ and its axis intercept $t_{s}$ in a system of Cartesian coordinates, i.e. $y=m_{s} \cdot x+t_{s}$. We can now take $m$ and $t$ as the axes of the parameter space and reformulate the line equation by $t_{s}=-m_{s} \cdot x+y$. Thus, each two-dimensional picture point $p=\left(x_{p}, y_{p}\right) \in \mathcal{D}$ in the picture space is mapped on a line $f_{p}$ with slope $-x_{p}$ and intercept $y_{p}$ in the parameter space, i.e. a line $f_{p}$ represented by $t=-m \cdot x_{p}+y_{p}$. The line $f_{p}$ in the parameter space models all linear segments (lines) that pass through $p$ in the original picture space. Thus, whenever several lines $f_{p_{1}}, \ldots, f_{p_{k}}$ in the parameter space intersect at a given point $\left(m_{i}, t_{i}\right) \in \mathcal{P}$, this indicates that the points $p_{1}, \ldots, p_{k} \in \mathcal{D}$ are located on a common line in picture space given by $y=m_{i} \cdot x+t_{i}$. A simplified example of the relationship between the picture space and the parameter space is visualized in Fig. 2. The three picture points $p_{1}, p_{2}$, and $p_{3}$ are located on a common line $s$ represented by $y=m_{s} \cdot x+t_{s}$ in the picture space (left). The corresponding mappings in the parameter space (right) $f_{p_{1}}, f_{p_{2}}$, and $f_{p_{3}}$ intersect at point $\left(m_{s}, t_{s}\right)$ in the parameter space. 

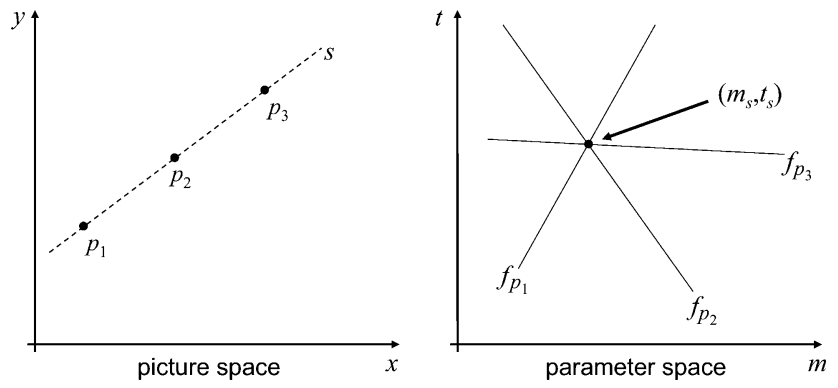

Fig. 2 Hough transform from picture space to parameter space using slope and intercept parameters.
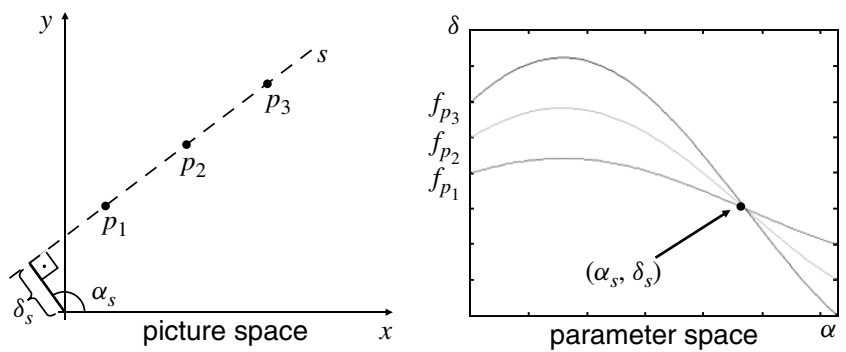

Fig. 3 Hough transform from picture space to parameter space using angle and radius parameters.

Obviously, both the slope and the intercept are unbounded which may cause some problems when applying this basic technique. Thus, [27] proposed to use spherical (also known as polar) coordinates, i.e. to use a parameter space based on angle and radius parameters rather than on slope and intercept parameters. The normal parameterization of a linear segment $s$ in two-dimensional is given by the angle $\alpha_{s}$ of its normal and its distance (radius) $\delta_{s}$ from the origin, i.e. $s$ is represented by $x \cdot \cos \alpha_{s}+y \cdot \sin \alpha_{s}=\delta_{s}$. If $\alpha_{s}$ is restricted to the interval $[0, \pi)$, the normal representation of a line is unique. The mapping from the picture space onto the parameter space using angle/radius works similar to the mapping using slope/intercept (see Figure 3). In either case, the parameter space represents all possible one-dimensional lines in the original two-dimensional data space.

In principle, each point of the data space is mapped on an infinite number of points in the parameter space which is not materialized as an infinite set, but instead as a trigonometric function in the parameter space. Each function in the parameter space represents all lines in the picture space crossing the corresponding point in data space. The intersection of two curves in the parameter space indicates a line through both the corresponding points in the picture space. The objective of a clustering algorithm is to find intersections of many curves in the parameter space representing lines through many database objects. The key feature of the Hough transform is that the distance of the points in the original data space is not considered any more.
Objects can be identified as associated to a common line even if they are far apart in the original feature space. As a consequence, the Hough transform is a promising candidate for developing a principle for subspace analysis that does not require the locality assumption and, thus, enables a global subspace-clustering approach.

In what follows, we will first present a novel principle for subspace analysis inspired by the ideas of the Hough transform (cf. Section 3.1). This principle enables us to transform the task of subspace clustering (in data space) into a grid-based clustering problem (in parameter space). Unlike grid-based methods operating directly in the data space, our method does not suffer from grid resolution and grid positioning problems. In order to perform this transformation, we first need to define the boundaries of the grid (cf. Section 3.2). Then we will show how to identify dense grid cells that represent potential subspace clusters (cf. Section 3.3). Since the parameter space is $d$ dimensional for a $d$-dimensional data space, finding dense grid cells becomes rather costly for higher-dimensional datasets. Thus, we will propose a more efficient search strategy for finding regions of interest in the parameter space (cf. Section 3.4). An important step in the clustering process is a recursive descent in order to find lower-dimensional clusters. We describe this descent in more detail in Section 3.5. We will also discuss how this recursive descent can be used to derive a hierarchy of subspace clusters (cf. Section 3.6). In fact, the clustering procedure implicitly provides a quantitative model of the reported clusters. We describe how this quantitative model can be made explicit in order to provide a comprehensive and user-friendly explanation of the detected clusters (cf. Section 3.7). Last but not least we will also summarize our subspace-clustering algorithm CASH (Clustering in Arbitrary Subspaces based on the Hough transform) and discuss some of its properties (cf. Section 3.8).

\subsection{Subspace Analysis: A Novel Principle}

Our novel principle for subspace analysis is based on a generalized description of spherical coordinates. Generalized spherical coordinates combine $d-1$ independent angles $\alpha_{1}, \ldots, \alpha_{d-1}$ with the norm $r$ of a $d$-dimensional vector $x=\left(x_{1}, \ldots, x_{d}\right)^{\top}$ to completely describe the vector $x$ with respect to the given orthonormal basis $e_{1}, \ldots, e_{d}$. We present a formalization analogously to [28]:

DEFINITION 1 (Spherical coordinates) Let $e_{i}, 1 \leq i \leq$ $d$, be an orthonormal basis in a $d$-dimensional feature space. Let $x=\left(x_{1}, \ldots, x_{d}\right)^{\top}$ be a $d$-dimensional vector on the hypersphere of radius $r$ with center at the origin. Let $u_{i}$ be the unit vector in the direction of the projection of vector $x$ onto the manifold spanned by $e_{i}, \ldots, e_{d}$. For the $d-1$ 
independent angles $\alpha_{1}, \ldots, \alpha_{d-1}$, let $\alpha_{i}, 1 \leq i \leq d-1$, be the angle between $u_{i}$ and $e_{i}$. Then the generalized spherical coordinates of vector $x$ are defined by:

$$
\begin{aligned}
x_{1} & =r \cdot \cos \left(\alpha_{1}\right) \\
x_{2} & =r \cdot \sin \left(\alpha_{1}\right) \cdot \cos \left(\alpha_{2}\right) \\
& \vdots \\
x_{i} & =r \cdot \sin \left(\alpha_{1}\right) \cdot \ldots \cdot \sin \left(\alpha_{i-1}\right) \cdot \cos \left(\alpha_{i}\right) \\
& \vdots \\
x_{d-1} & =r \cdot \sin \left(\alpha_{1}\right) \cdot \ldots \cdot \sin \left(\alpha_{d-2}\right) \cdot \cos \left(\alpha_{d-1}\right) \\
x_{d} & =r \cdot \sin \left(\alpha_{1}\right) \cdot \ldots \cdot \sin \left(\alpha_{d-2}\right) \cdot \sin \left(\alpha_{d-1}\right)
\end{aligned}
$$

Generally:

$$
x_{i}=r \cdot\left(\prod_{j=1}^{i-1} \sin \left(\alpha_{j}\right)\right) \cdot \cos \left(\alpha_{i}\right),
$$

where $\alpha_{d}=0$.

For any point $p \in \mathcal{D} \subseteq \mathbb{R}^{d}$ there exists an infinite number of hyperplanes containing $p$. The spherical coordinates are utilized to define the normal vector of the Hessian normal form for any of those hyperplanes, i.e. each hyperplane is uniquely defined by a point $p$ and $d-1$ angles $\alpha_{1}, \ldots, \alpha_{d-1}$, with $\alpha_{i} \in[0, \pi)$, defining the normal vector. Thus, any point $p$ together with any tuple of angles $\alpha_{1}, \ldots, \alpha_{d-1}$, can be mapped by the following parameterization function to the distance of the corresponding hyperplane to the origin.

DEFINITION 2 (Parameterization Function) Let $p=$ $\left(p_{1}, \ldots, p_{d}\right)^{\top} \in \mathcal{D} \subseteq \mathbb{R}^{d}$ be a $d$-dimensional vector, and let $n=\left(n_{1}, \ldots, n_{d}\right)^{\top}$ be a $d$-dimensional unit vector specified by $d-1$ angles $\alpha_{1}, \ldots, \alpha_{d-1}$ according to Definition 1 . Then the parameterization function $f_{p}: \mathbb{R}^{d-1} \rightarrow \mathbb{R}$ of vector $p$ denotes the distance of the hyperplane defined by the point $p$ and the normal vector $n$ to the origin:

$$
\begin{aligned}
f_{p}\left(\alpha_{1}, \ldots, \alpha_{d-1}\right) & =\langle p, n\rangle \\
& =\sum_{i=1}^{d} p_{i} \cdot\left(\prod_{j=1}^{i-1} \sin \left(\alpha_{j}\right)\right) \cdot \cos \left(\alpha_{i}\right)
\end{aligned}
$$

Based on Definition 2, we can map any point $p \in \mathbb{R}^{d}$ to a function in a $d$-dimensional parameter space $\mathcal{P}$ representing all possible hyperplanes containing $p$. This parameter space is spanned by the $d-1$ angles $\alpha_{1}, \ldots, \alpha_{d-1}$ of the normal vectors defining the hyperplanes in Hessian normal form and their distances $\delta=f_{p}\left(\alpha_{1}, \ldots, \alpha_{d-1}\right)$ to the origin.

By means of the parameterization function (Definition 2), we can also extend the properties of the original Hough transform as stated in [27] for the mapping of twodimensional points to $d$-dimensional data spaces and the corresponding parameter spaces:

PROPERTY 1: A point $p \in \mathcal{D} \subseteq \mathbb{R}^{d}$ in data space is represented by a sinusoidal curve $f_{p}: \mathbb{R}^{d-1} \rightarrow \mathbb{R}$ in parameter space $\mathcal{P}$.

Figure 4 illustrates a three-dimensional example of this property. Three points $p_{1}, p_{2}$, and $p_{3}$ in data space are mapped onto the corresponding sinusoidal curves $f_{p_{1}}, f_{p_{2}}$, and $f_{p_{3}}$, respectively, in parameter space.

PROPERTY 2: A point $\left(\alpha_{1}, \ldots, \alpha_{d-1}, \delta\right) \in \mathcal{P}$ in parameter space corresponds to a $(d-1)$-dimensional hyperplane in data space.

In Fig. 4 , the point $\left(\alpha_{1}^{s}, \alpha_{2}^{s}, \delta^{s}\right)$ in parameter space represents the two-dimensional plane $s$ with

$$
\begin{gathered}
\delta^{s}=\cos \left(\alpha_{1}^{s}\right) \cdot x_{1}+\sin \left(\alpha_{1}^{s}\right) \cdot \cos \left(\alpha_{2}^{s}\right) \cdot x_{2} \\
+\sin \left(\alpha_{1}^{s}\right) \cdot \sin \left(\alpha_{2}^{s}\right) \cdot x_{3}
\end{gathered}
$$

in data space.

PROPERTY 3: Points that are located on a $(d-1)-$ dimensional hyperplane in data space correspond to sinusoidal curves through a common point in parameter space.

The three points $p_{1}, p_{2}, p_{3} \in \mathcal{D}$ (Fig. 4) are located on the two-dimensional plane $s$. Their corresponding sinusoidal curves $f_{p_{1}}, f_{p_{2}}, f_{p_{3}}$ intersect in the point $\left(\alpha_{1}^{s}, \alpha_{2}^{s}, \delta^{s}\right) \in \mathcal{P}$, where $\alpha_{1}^{s}, \alpha_{2}^{s}$ and $\delta^{s}$ are the parameters of plane $s$ as given above (cf. Property 2).

PROPERTY 4: Points located on the same sinusoidal curve in parameter space represent $(d-1)$-dimensional hyperplanes through the same point in data space.

For example, in Fig. $4, f_{p_{1}}$ in parameter space represents all two-dimensional planes through $p_{1}$ in data space. Thus, any point on $f_{p_{1}}$ in parameter space represents a given two-dimensional plane in data space that passes through $p_{1}$.

Properties 1-4 induce that an intersection point in the parameter space indicates points in the data space that are located on a common $(d-1)$-dimensional hyperplane. In order to detect those linear hyperplanes in the data space, 


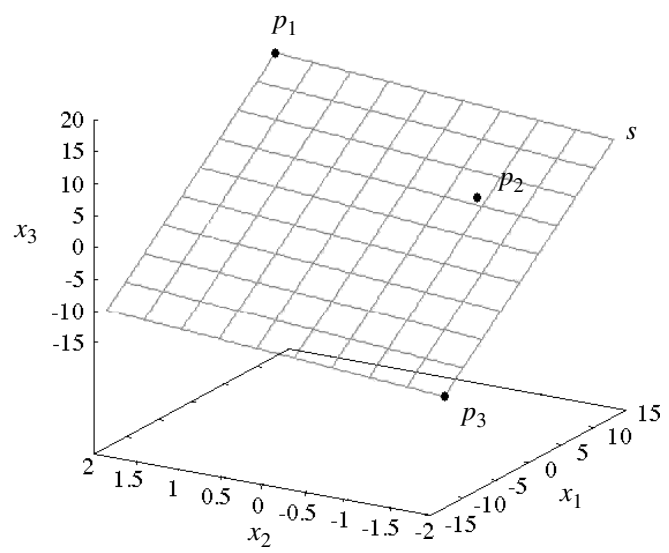

(a) Three points $p_{1}, p_{2}, p_{3}$ on a plane $s \subseteq \mathbb{R}^{3}$.

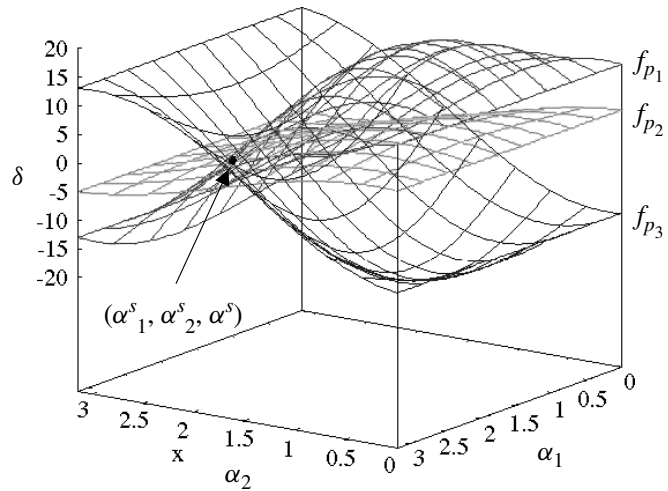

(b) Corresponding parametrization functions.

Fig. 4 Transform of a three-dimensional data space into a three-dimensional parameter space.

the task is to search for points in the parameter space where many sinusoidal curves intersect. Since computing all possibly interesting intersection points is computationally too expensive, we discretize the parameter space by some grid and search for grid cells with which many sinusoidal curves intersect. For that purpose, for each grid cell the number of intersecting sinusoidal curves is aggregated. Owing to this discretization of the parameter space, exact intersections are no longer considered. Rather, a slight impreciseness is allowed while modeling a certain degree of jitter given by the grid resolution. The higher the grid resolution is, the lower is the allowed degree of jitter, i.e. the more accurate the recognition of the line segments.

With the proposed concepts, we transform the original subspace-clustering problem (in data space) into a gridbased clustering problem (in parameter space).

\subsection{Specifying the Boundaries of the Grid}

To define a discretization of the parameter space, the range of the axes must be known. The axes for the angleparameters $\alpha_{1}, \ldots, \alpha_{d-1}$, are bounded by $[0, \pi)$. The $\delta$ axis ranges from the minimum of all minima of all parameterization functions to the maximum of all their maxima within $[0, \pi)^{d-1}$. Each $f_{p}$ is a sinusoid with a period of $2 \pi$. Thus, any $f_{p}$ has exactly one global extremum in the interval $[0, \pi)^{d-1}$. If the extremum of $f_{p}$ is a maximum, the minimal value for $f_{p}$ in the given interval has to be determined and vice versa.

To find the global extremum of a parameterization function $f_{p}$ in the interval $[0, \pi)^{d-1}$, those angles $\alpha_{1}, \ldots$, $\alpha_{d-1}$ need to be determined where all the first-order derivatives of $f_{p}$ are zero, and the Hessian matrix of $f_{p}$ is either positive or negative definite. As noted above, $f_{p}$ is guaranteed to have exactly one global extremum $f_{p}\left(\tilde{\alpha}_{1}, \ldots, \tilde{\alpha}_{d-1}\right)$ in $[0, \pi)^{d-1}$. The values for the angles $\tilde{\alpha}_{n}(n=1, \ldots, d-1)$ of the global extremum of $f_{p}$ are given by (cf. Appendix A for details):

$$
\tilde{\alpha}_{n}=\arctan \left(\frac{\sum_{j=n+1}^{d} p_{j} \cdot\left[\prod_{k=n+1}^{j-1} \sin \left(\tilde{\alpha}_{k}\right)\right] \cdot \cos \left(\tilde{\alpha}_{j}\right)}{p_{n}}\right)
$$

Given the global extremum of a parameterization function $f_{p}$ in the interval $[0, \pi)^{d-1}$, we have to distinguish several cases to determine the opposite value, i.e. to determine the maximum of $f_{p}$ if the global extremum of $f_{p}$ is a minimum or to determine the minimum of $f_{p}$ if the global extremum is a maximum. In the following text, we describe how to determine the point $\alpha^{\text {min }}$ where the parameterization function $f_{p}$ has a minimum in interval $[0, \pi)^{d-1}$ given that the global extremum is a maximum. In the opposite case, the point $\alpha^{\max }$ where the parameterization function $f_{p}$ has a maximum in interval $[0, \pi)^{d-1}$ given the global extremum is a minimum can be determined analogously. Please refer to Appendix B for a detailed formalization of this step.

We determine the point $\alpha^{\min }=\left(\alpha_{1}^{\min }, \ldots, \alpha_{d-1}^{\min }\right)$ where the parameterization function $f_{p}$ has a minimum in interval $[0, \pi)^{d-1}$ as follows: First, the angle $\bar{\alpha}_{d-1}$ on axis $(d-1)$ is determined where $f_{p}$ has an extremum on this axis. Dependent on the type of the extremum in $\bar{\alpha}_{d-1}$ and the location of $\bar{\alpha}_{d-1}$ in the interval $[0, \pi)$, the minimum angle $\alpha_{d-1}^{\min }$ on axis $(d-1)$ in interval $[0, \pi)$ is determined. In the next step, axis $(d-2)$ will be considered: Now, the angle $\bar{\alpha}_{d-2}$ will be determined, where $f_{p}$ has an extremum on this axis under the constraint of the known minimum on axis $d-1$, which is given by $\alpha_{d-1}^{\min }$. Analogously to the first step, dependent on the type of the extremum in $\bar{\alpha}_{d-2}$ and the location of $\bar{\alpha}_{d-2}$ in the interval $\left[\check{\alpha}_{d-2}, \hat{\alpha}_{d-2}\right)$, the minimum 
angle $\alpha_{d-2}^{\min }$ is determined. In this way, all minimum angles are determined under the constraint of the known minima on the already processed axes.

In summary, given for each parameterization function $f_{p}$ its minimal and maximal value $\alpha_{p}^{\min }$ and $\alpha_{p}^{\max }$ in interval $[0, \pi)^{d-1}$, the $\delta$-axis of the parameter space $\mathcal{P}$ is bounded by $\left[\delta_{\min }, \delta_{\max }\right]=\left[\min _{p \in \mathcal{D}}\left(f_{p}\left(\alpha_{p}^{\min }\right)\right), \max _{p \in \mathcal{D}}\left(f_{p}\left(\alpha_{p}^{\max }\right)\right)\right]$ and $\mathcal{P}=\left[\delta_{\min }, \delta_{\max }\right] \times[0, \pi)^{d-1}$.

\subsection{Identifying Dense Grid Cells}

Given a discretized parameter space, now those grid cells (hypercuboids) have to be found that are intersected by parameterization functions of a minimum number $m$ of functions. Hypercuboids containing at least $m$ parameterization functions are called dense regions of the parameter space. Those dense regions represent arbitrarily oriented subspaces in the data space accommodating at least $m$ points. This is illustrated in Fig. 5. The two subspace clusters forming lines in the data space (cf. Fig. 5(a)) are represented by two distinct dense regions in the parameter space (cf. Fig. 5(b)).

To find those dense regions in the parameter space, for each grid cell or hypercuboid, the number of parameterization functions which intersect this hypercuboid has to be counted. This can be done conveniently by determining those values $\alpha_{p}^{\min }$ and $\alpha_{p}^{\max }$ in a given interval $[\check{\alpha}, \hat{\alpha}) \subseteq[0, \pi)^{d-1}$ that minimize and maximize a parameterization function $f_{p}$. Then, all hypercuboids based on this interval and positioned between $f_{p}\left(\alpha_{p}^{\min }\right)$ and $f_{p}\left(\alpha_{p}^{\max }\right)$ are intersected by $f_{p}$. The values $\alpha_{p}^{\min }$ and $\alpha_{p}^{\max }$ in a given interval $[\check{\alpha}, \hat{\alpha}) \subseteq[0, \pi)^{d-1}$ that minimize and maximize $f_{p}$ can be determined analogously to the algorithm specified in Section 3.2 where the given interval was assumed to be $[0, \pi)^{d-1}$.

\subsection{Efficiently Finding Regions of Interest}

A region qualifying as a dense region, but containing exclusively one cluster, possibly needs to be defined by a rather small interval of angles and also a rather small interval of distances from the origin because otherwise the same interval could also contain functions representing points of other clusters (cf. the dense region of cluster $\mathrm{C} 1$ in Fig. 5). For that purpose, a rather high number of intervals in each dimension of the parameter space is needed, resulting in a huge number of grid cells possibly qualifying as dense regions. Thus, searching the parameter space with a predefined grid in the range $[0, \pi)$ for each angle and $\left[\delta_{\min }, \delta_{\max }\right]$ for the distance from the origin is not feasible for high-dimensional data in terms of space and time complexity.

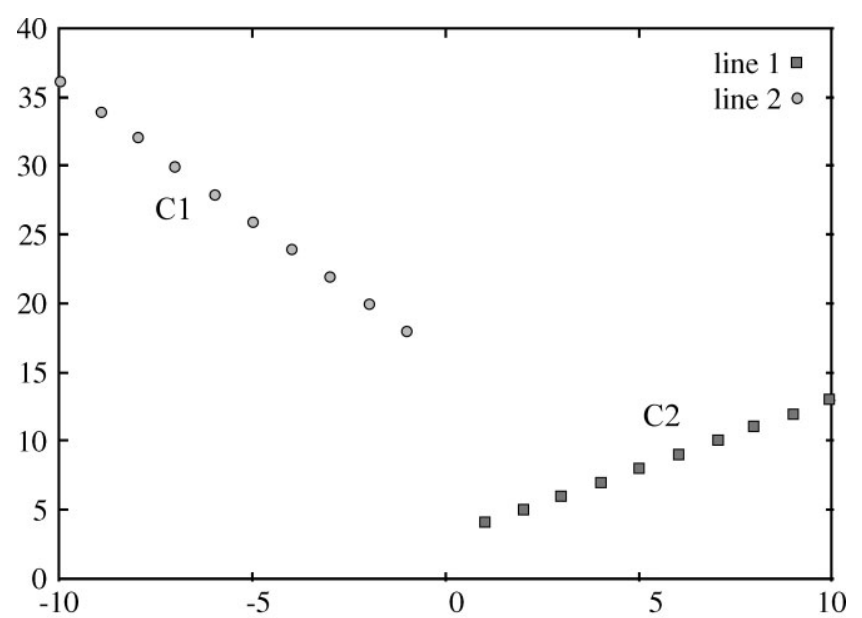

(a) Two lines in data space

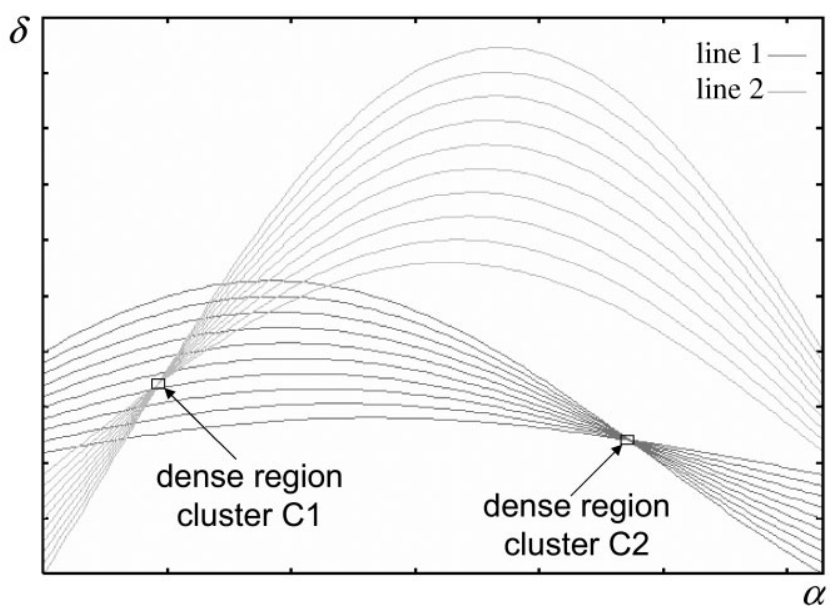

(b) Dense regions in parameter space

Fig. 5 Dense regions in parameter space capturing two lines in data space.

To avoid exponential complexity, the following search strategy for the parameter space is proposed:

(1) The axes (distance and angles) are divided successively in a static order given by $\delta, \alpha_{1}, \ldots, \alpha_{d-1}$. After dividing one axis, from the resulting two hypercuboids the one containing most points is selected for refinement. If both hypercuboids contain an equal number of points, the first one is selected (arbitrarily). The selected hypercuboid is divided recursively by splitting the next axis. The neglected hypercuboid is kept in a queue.

(2) If both children of a divided hypercuboid contain less than $m$ points, the search in the corresponding path is discontinued. Unless the queue is empty, the next hypercuboid in the queue is examined using the same procedure. In the queue, hypercuboids are ordered descendingly by the number of points contained by a hypercuboid. If two hypercuboids contain an equal number of points, the smaller one 
is preferred, since a smaller interval containing an equal number of data points is a more promising candidate.

(3) At a predefined depth (i.e. a given number $s$ of successive splits), a hypercuboid (i.e. the corresponding interval) is considered to be sufficiently small to define a hyperplane containing a subspace cluster. If the number of points within the hypercuboid exceeds a predefined number $m$ of points, these points are considered to build a subspace cluster. The corresponding subspace is treated as a new data space containing all the points accounted for in the hypercuboid. This new data space of dimensionality $d-1$ undergoes the same procedure recursively, while $d>2$, i.e. CASH is called for the points in the hypercuboid using the corresponding subspace as data space (see Section 3.5 for a more detailed explanation of the recursive descent). If no subspace cluster of lower dimensionality is found in this $(d-1)$-dimensional space, all the points in this subspace are supposed to build a $(d-1)$-dimensional subspace cluster.

(4) All points participating at a $(d-1)$-dimensional subspace cluster derived at a search path are removed from the $d$-dimensional data space. The queue is reorganized and hypercuboids are removed if they now contain less than $m$ points. A new search path based on the next hypercuboid in the queue is pursued.

(5) The search is complete, if in the $d$-dimensional space no interval is found containing at least $m$ points.

This search strategy determines clusters of at least $m$ points in any arbitrarily oriented subspace, and provides a description with an accuracy regarding the orientation $\alpha$ and the distance $\delta$ from the origin as defined by the predefined number $s$ of splits.

Unlike traditional grid-based clustering approaches, CASH has no problems if a region of interest (i.e. a cluster) is located at the boundary of two connected grid cells, $g_{1}$ and $g_{2}$. In that case, the functions will intersect both neighboring grid cells and both grid cells, $g_{1}$ and $g_{2}$, will be dense. CASH will refine one of these grid cells (e.g. $g_{1}-$ cf. step 1) until the cluster is found. After that, CASH eliminates the participating points (i.e. functions) and, thus, the second grid cell $\left(g_{2}\right)$ will not be dense anymore (step 4).

Owing to the recursive search in an obtained cluster (step 3), a cluster hierarchy is gained along the way, i.e. a subspace cluster may in turn contain nested subspace clusters of lower dimensionality. In that case, it may be interesting to report all nested clusters and the information of the 'contained-in' relationships. Section 3.6 provides more details on how such a hierarchy can be obtained.

\subsection{Recursive Descent}

In this section, we describe in more detail the recursive procedure to find lower-dimensional clusters within higherdimensional clusters as mentioned in step 3 of the search heuristic (Section 3.4).

If CASH finds a cluster, i.e. a $d$-dimensional hypercuboid $g(d>2)$ at a predefined depth (i.e. a given number $s$ of successive splits) being sufficiently dense, the search space is transformed according to the current orientation and affinity of the subspace defined by the hypercuboid $g$. In particular, the hypercuboid $g$ defines a subspace by means of the Hessian normal form with a certain error (as defined by the intervals of angles $\left[\check{\alpha}_{i}, \hat{\alpha}_{i}\right)$ for each axis $i$ and the interval of distances $\left[\delta_{\min }, \delta_{\max }\right]$ from the origin spanned by $g)$. The corresponding hyperplane (a $(d-1)$-dimensional affine subspace) is given by spherical coordinates of the normal vector $n$ assuming the mean of $\delta_{\min }$ and $\delta_{\max }$ as the length (radius $r$ ) of $n$ and for each angle the mean of the corresponding values of $\check{\alpha}$ and $\hat{\alpha}$, respectively. In other words, the spherical coordinates of $n$ are given by

$$
r=\frac{\delta_{\min }+\delta_{\max }}{2}
$$

and

$$
\alpha_{i}=\frac{\check{\alpha}_{i}+\hat{\alpha}_{i}}{2}
$$

(for $1 \leq i \leq d-1$ ). The Cartesian coordinates are given as in Definition 1 by

$$
x_{i}=r \cdot\left(\prod_{j=1}^{i-1} \sin \left(\alpha_{j}\right)\right) \cdot \cos \left(\alpha_{i}\right) .
$$

The normal vector $n$ is then completed to an orthonormal basis by adding $d-1$ linear independent arbitrary basis vectors (which is generally possible in a $d$-dimensional space). The corresponding orthonormal matrix $N$ facilitates the transformation of the parameterization functions from the $d$-dimensional space into the $(d-1)$-dimensional subspace by multiplication with $N$ and projection onto the space given by $N \backslash n$. This way, a new, $(d-1)$ dimensional subspace is defined. The dataset corresponding to this subspace contains only the parameterization functions intersecting the hypercuboid $g$. For the next step, CASH is applied to the points of the cluster represented by $g$ transformed into the new $(d-1)$-dimensional subspace. In each next step the search space is therefore reduced in dimensionality and at least not increased with respect to the number of database objects. 


\subsection{Deriving a Hierarchy of Subspace Clusters}

By means of the recursive descent, CASH directly yields a hierarchy of arbitrarily oriented subspace clusters. All points belonging to a dense $(d-1)$-dimensional grid cell also belong to the $d$-dimensional grid cell that has been previously analyzed in order to find lower-dimensional clusters. Thus, when recursively descending after identifying a cluster, we simply have to store a pointer from the higherdimensional cluster to the lower-dimensional cluster. As a result, we get a containment hierarchy of clusters and their corresponding subspaces. This hierarchy displays an important relationship among clusters. If any $l$-dimensional cluster $A$ is contained in a $k$-dimensional cluster $B(l<k)$ according to this relationship, this means that all points of cluster $A$ are not only located on a common $l$-dimensional cluster hyperplane but also located on the $k$-dimensional cluster hyperplane that is shared by the points in $B$. Cluster $B$ can thus be regarded as a superset of $A$. A higherdimensional superset $B$ of a cluster $A$ can be regarded as an interesting cluster itself, if $|B-A| \geq m$.

From the point of view of a hierarchy of subspaces, the difference between the points in $B-A$ and the points in $B$ is that points in $B-A$ exhibit a correlation not only among the $l$ attributes that are correlated for the points in $B$, but also among $k-l$ additional attributes. Knowing these relationships is quite interesting when evaluating and interpreting the reported clusters in order to find hidden causalities in the data.

The hierarchy can be visualized as a tree. Each node of a tree represents a cluster. The root node (level 0) of the tree represents the entire database forming a 'dummy' $d$-dimensional cluster in which all other 'true' clusters are contained. A node at level $k$ represents a $(d-k)$ dimensional cluster. An edge between a $k$ - and an $l$ dimensional cluster $(l<k)$ represents the containment of the $l$-dimensional cluster within the $k$-dimensional one. Finally, any node on level $l \geq 1$ without a parent node is linked to the root.

\subsection{Deriving a Cluster Model}

In [29] a PCA-based method for deriving quantitative models of correlation clusters is proposed. The presented model of a cluster mainly consists of an equation system that quantitatively reveals the relationships between the participating attributes. This model is derived by applying PCA to the points in the cluster and then identifying the major and minor principal axis of the point set using some thresholds.

Applying the method proposed in [29] is of course generally possible, but our algorithm CASH provides such a model implicitly. In particular, the model is defined by means of the subspace that is specified by the corresponding grid cell as discussed in Section 3.5. As a consequence, rather than applying a complex (and possibly misleading) extra procedure, we can directly derive for each cluster found a model describing the subspace accommodating the cluster. This is a clear benefit because the method in [29] relies on a completely different concept (i.e. PCA) and employs some heuristic thresholds. Thus, it may produce a model that represents a subspace different from that represented by the grid cell found by $\mathrm{CASH}$.

As explained above, the subspace of a cluster (i.e. the cluster hyperplane) is represented by a hypercuboid $g$. This hypercuboid represents the spherical coordinates of the normal vector of the cluster hyperplane. The transformations performed for the recursive descent (cf. Section 3.5) are injective operations followed by a projection. The projection results in an error of a predefined maximal margin (given by the perimeter of the corresponding grid cell in angles and radius). Thus, performing the inverse operations results in a simplified but concise model describing the orientation, affinity, and dimensionality of the subspace accommodating the cluster members. In order to derive an equation system similar to the cluster model proposed in [29], we have to transform the spherical coordinates of all normal vectors that have been generated during the recursive descent into Cartesian coordinates. For a $l$-dimensional cluster we thereby obtain $(d-l)$ normal vectors and, consequently, a system of $(d-l)$ equations.

In order to exemplify these ideas, assume that we have obtained a $(d-1)$-dimensional cluster $C$ in the first step of CASH by identifying a dense grid cell $g^{C}$. As mentioned earlier, the hypercuboid $g^{C}$ defines a subspace by means of the Hessian normal form with a certain error (as defined by the intervals of angles $[\check{\alpha}, \hat{\alpha})$ for each axis and the interval of distances $\left[\delta_{\min }, \delta_{\max }\right]$ from the origin spanned by $g^{C}$ ). The corresponding hyperplane (a $d-1$-dimensional affine subspace) is given by spherical coordinates of the normal vector $n^{C}$ that can again be derived as defined in Eqs. 1 and 2. As described above, we can transform these coordinates into Cartesian coordinates [cf. Eq. 3] and derive an equation system with $(d-1)$ free attributes, i.e. the equation system provides one equation for each normal vector, e.g.

$$
a_{1} x_{1}+\cdots+a_{d-1} x_{d-1}+a_{d} x_{d}=0
$$

In this equation, the coefficients $a_{i}$ are the Cartesian coordinates of the normal vectors. In our example, this would provide a quantitative model describing a correlation cluster of correlation dimensionality $(d-1)$ (corresponding to the number of free attributes). The usefulness of such models for the interpretation of correlation clusters has been demonstrated in [29]. In particular, the quantities 
of the relationships can directly be derived by the coefficients $a_{1}, \ldots, a_{d}$. In addition, in this example, we can also directly deduce that all attributes with $a_{i} \neq 0$ are involved in the correlation.

\subsection{Properties of the Algorithm}

The algorithm CASH transforms the data objects from $\mathcal{D} \subseteq \mathbb{R}^{d}$ into a corresponding parameter space (based on radius and angles) $\mathcal{P}=\left[\delta_{\min }, \delta_{\max }\right] \times[0, \pi)^{d-1}$. After that, CASH identifies dense regions in that parameter space using the search strategy proposed above. These dense regions represent arbitrarily oriented subspace clusters in the data space. For each dense region, a recursive descent is initialized. The resulting hierarchy of subspace clusters is visualized by a tree structure placing the complete database in the root of the tree representing the entire database.

\subsubsection{Complexity.}

Let $N$ be the number of data points in a $d$-dimensional data space. When bisecting the parameter space of $\alpha_{i}$ and $\delta$, we need to determine those database points, whose parameter functions intersect with the generated cells in the parameter space. This is done by the maximization and minimization of $\delta$ given the constraints on $\alpha_{i}$ (i.e. $\check{\alpha_{i}} \leq \alpha_{i}<\hat{\alpha_{i}}$ for all $\left.1 \leq i \leq d-1\right)$ requiring $O\left(d^{3}\right)$ time per object and cell.

The CASH algorithm performs a recursive bisection of the data space where all bisections with fewer than $m$ associated database points are discarded. Since bisection cells which do not belong to any cluster are only randomly associated with a few arbitrary points, the bisection process for those cells stops at a high level of the bisection tree. Only cells belonging to actual subspace clusters are bisected until the defined maximum number $s$ of bisection levels is reached. Therefore, for a dataset containing $c$ clusters, a number $O(s \cdot c)$ of nodes in the bisection tree are encountered, each causing $O\left(N \cdot d^{3}\right)$ work to find all subspace clusters. Together, we have an average time complexity in $O\left(s \cdot c \cdot N \cdot d^{3}\right)$. Let us note, though, that the performance can deteriorate considerably in the worst case. If all cells are dense on all but the last level, all paths have to be searched and the complexity is essentially the same as the complexity of a complete enumeration approach, $O\left(2^{d}\right)$.

\subsubsection{Input parameters.}

CASH requires the user to specify two input parameters: The first parameter $m$ specifies the minimum number of sinusoidal curves that need to intersect a hypercuboid in the parameter space such that this hypercuboid is regarded as a dense area. Obviously, this parameter represents the minimum number of points in a cluster, and thus, is very intuitive. The second parameter $s$ specifies the maximal number of splits along a search path (split-level). Thus, it controls the maximal allowed deviation from the hyperplane of the cluster in terms of orientation and jitter. We show in our experiments, that CASH is rather robust with respect to $s$. Since CASH does not require parameters that are hard to guess like the number of clusters, the average dimensionality of the subspace clusters, or the size of the Euclidean neighborhood based on which the similarity of the subspace clusters is learned, it is much more usable and stable than its competitors.

\subsubsection{Alternative parameterization.}

It is also possible to treat the split-level for the radius and the angles separately. This increases the number of parameters by 1 , but allows to treat different kinds of deviations from the idealized cluster hyperplane differently: The allowed variance in the radius corresponds to the allowed thickness of the hyperplane, i.e. the tolerated deviation of cluster members orthogonally from the hyperplane. This kind of error is usually encountered in real-world datasets. The tolerated variance in the angles corresponds to the tolerated variance in the orientation of the hyperplane. A larger allowance here makes it possible to grasp clusters where the members do not follow a perfectly linear correlation.

\section{EVALUATION}

\subsection{Efficiency}

To evaluate the scalability of CASH with respect to the size of the dataset, we created 10 datasets containing 4 , equally sized one-dimensional clusters in a five-dimensional data space with an increasing number of points ranging from 10000 to 100000 . CASH performs comparably well to ORCLUS. Both outperform 4C significantly (cf. Fig. 6). As a fair setting, we gave as parameter $k$ to ORCLUS the exact number of clusters in the dataset (i.e. $k=4$ ), and parameter $l$ has been set to the correct correlation dimensionality of the clusters (i.e. $l=1$ ). For $4 \mathrm{C}$, the parameters have been set to $k=\mu=100, \varepsilon=0.1, \lambda=1$, and $\delta=0.01$, reflecting the actual cluster structure in the synthetic datasets. The parameter setting for CASH was $s=40$ and $m=2500$.

To assess the impact of the dimensionality of the data space on the runtime of $\mathrm{CASH}$, we created 10 datasets ranging in dimensionality from 5 to 50 , each dataset containing a one-dimensional cluster of 10,000 points. The parameters were set to $s=50$ and $m=5000$. Figure 7 shows the scalability of CASH logarithmically on both axes, dimensionality and runtime. The graph is a line with 


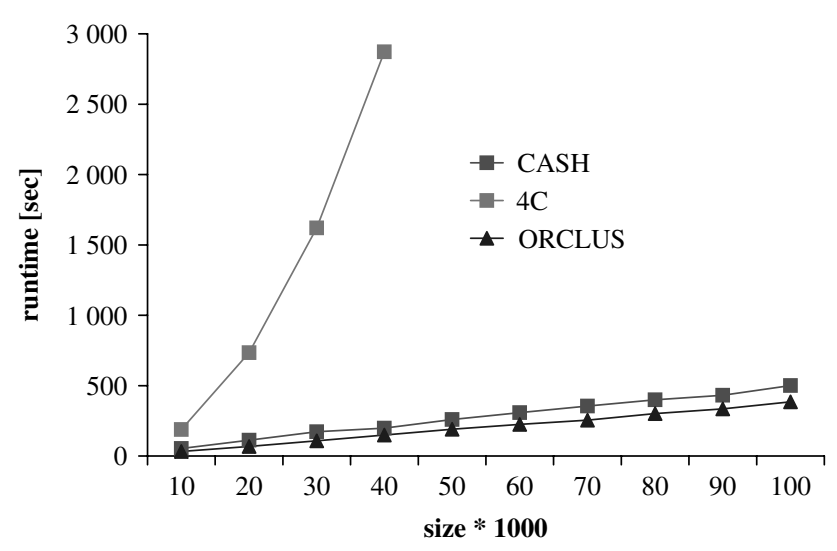

Fig. 6 Scalability with respect to size.

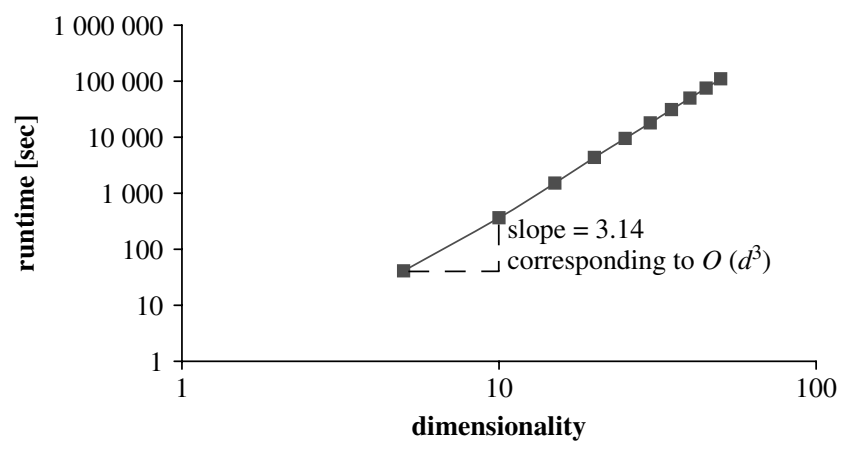

Fig. 7 Scalability of CASH with respect to dimensionality.

slope 3.14 , approximately corresponding to the expected runtime behavior.

In both test scenarios, the objective was to find onedimensional clusters in a $d$-dimensional data space, since this is the most complex task for CASH, requiring a maximal recursive descent from $d-1$ until subspace dimensionality 1 is reached.

\subsection{Effectiveness}

The parameter $s$ clearly influences the runtime behavior to a certain degree. However, CASH reaches satisfying behavior in terms of effectiveness for even relatively low values for $s$. Figure 8 illustrates the effect of $s$ on runtime and effectiveness simultaneously. On a five-dimensional dataset containing two one-dimensional clusters, each containing 500 points, and 500 points of noise, $\mathrm{CASH}$ reaches an $F$-measure of $100 \%$ already for $s=35$, while the runtime remains relatively low with $3.29 \%$ compared to the maximum runtime for $s=75$.

To assess the robustness of CASH against noise, we created ten datasets consisting of correlation clusters based on linear dependencies among subsets of attributes. As discussed in [29], linear dependencies among subsets

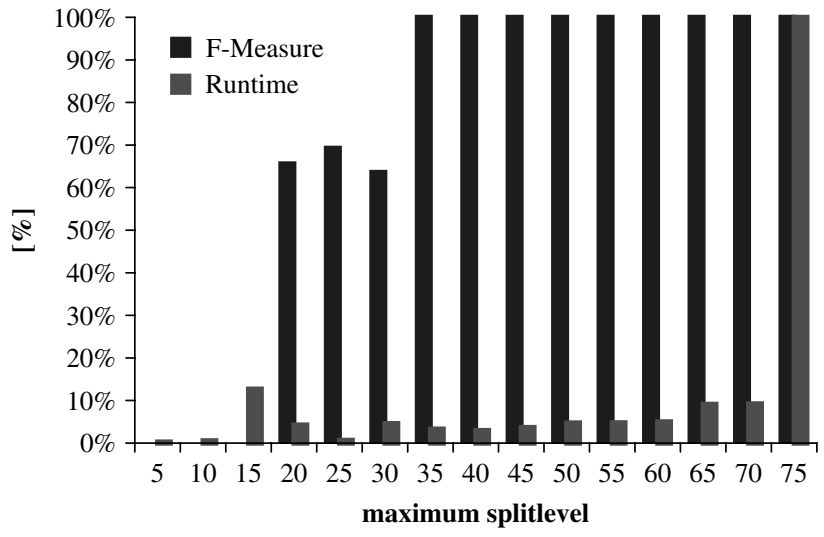

Fig. 8 F-measure and runtime of CASH with respect to maximum split-level.

of attributes are characteristic of correlation clusters. The datasets contain an increasing level of noise objects ranging from 0 to $90 \%$ of the complete dataset. Thus, for these datasets, each point belongs to a predefined cluster or noise. The clusters and the noise set can be regarded as classes. The algorithms can thus be evaluated in a supervised setting.

Figure 9 shows the comparison in robustness with ORCLUS and $4 \mathrm{C}$. The parameter setting for ORCLUS has been $l=1$ and $k=2$, reflecting the true number of clusters and their dimensionality. For $4 \mathrm{C}$, the optimal parameter setting has been used with $k=\mu=5, \varepsilon=0.12, \lambda=1$, and $\delta=0.01$. For CASH, the parameters have been chosen as $s=30$ and $m=50$. Let us note that CASH did not require any efforts for optimization of parameter settings. While both 4C and ORCLUS performed relatively well for very low levels of noise objects, their performance deteriorates for a higher degree of noise. CASH remains constantly on an $F$-measure of $100 \%$ up to a noise level of $80 \%$. Even for an extremely high level of noise $(90 \%), \mathrm{CASH}$ still reaches an $F$-measure of $94 \%$.

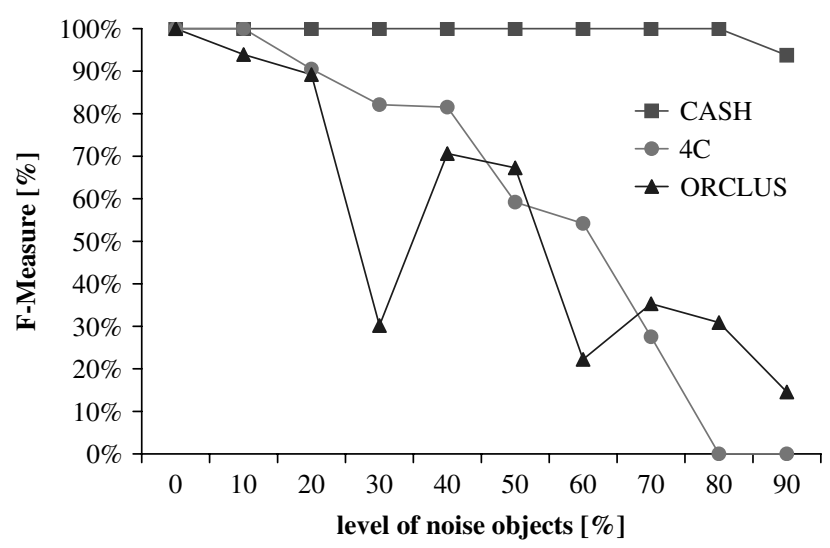

Fig. 9 F-Measure with respect to noise level. 


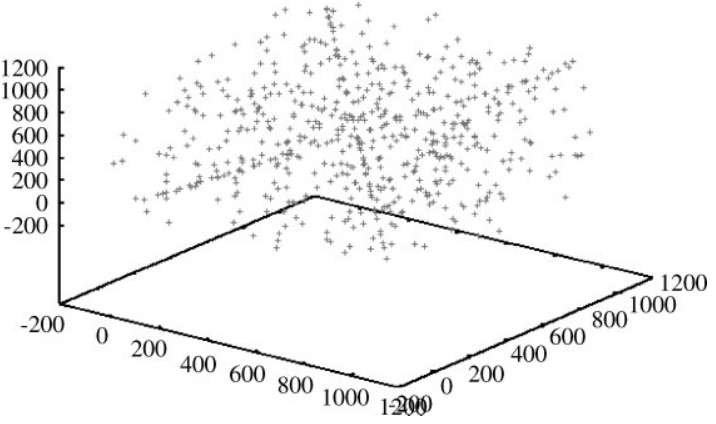

(a) Synthetic data set DS1.

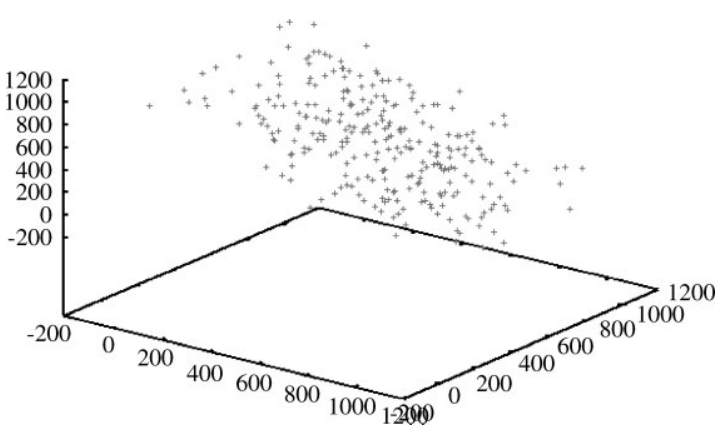

(c) ORCLUS - Cluster 1.

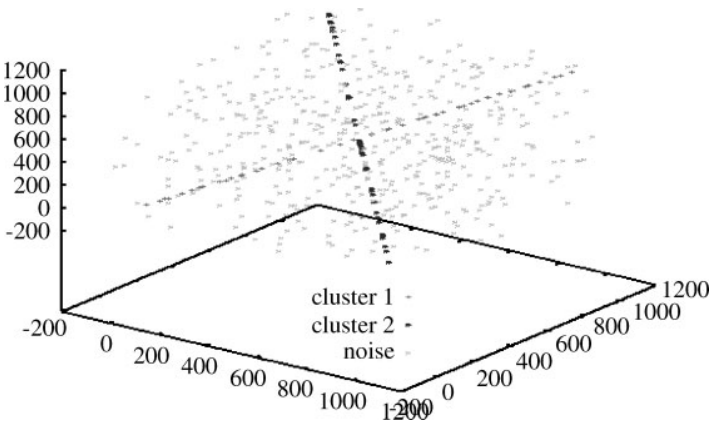

(b) $\mathrm{CASH}$ - Clustering.

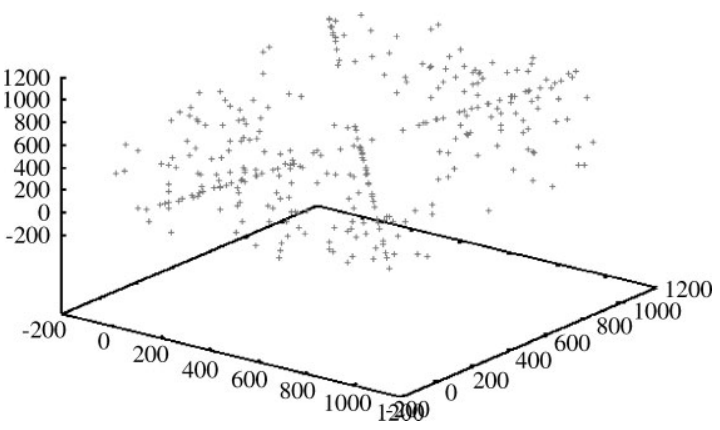

(d) ORCLUS - Cluster 2.

Fig. 10 Clustering synthetic dataset DS1.

We illustrate the robustness of CASH against noise on an exemplary three-dimensional dataset depicted in Fig. 10(a). $\mathrm{CASH}$ finds the two one-dimensional subspace clusters (each of size 50) embedded in 500 noise points exactly (cf. Fig. 10(b)). The results of ORCLUS (with optimal parameter setting $l=1$ and $k=2$ ) are shown in Figs 10(c) and 10(d). As it can be seen, the clusters found by ORCLUS do not reflect the real cluster structure at all. For $4 \mathrm{C}$, we tried several parameter settings. Unfortunately, 4C was never able to find a meaningful cluster structure at all.

Further experiments on high-dimensional datasets have been performed with CASH, 4C, and ORCLUS. The datasets contained complex subspace cluster structures with sparse clusters, including subspace clusters of significantly differing dimensionality, subspace clusters hierarchically embedded in higher-dimensional subspaces, and noise objects. In none of the performed experiments were $4 \mathrm{C}$ or ORCLUS able to find meaningful clusters, while CASH exactly detected the cluster structures in most cases. As an example, we present the results on a complex threedimensional dataset shown in Fig. 11(a), containing three one-dimensional clusters each of 500 points, two twodimensional planes each containing 500 points, and 500 points of noise. One of the planes is intersected by two lines, the other plane is intersected by one line. Here, CASH is able to identify the cluster structure of all five clusters exactly (Fig.11(b)). In contrast, 4C (cf. Fig.11(c), parameters optimized to $k=\mu=20, \varepsilon=0.1, \lambda=2$, and $\delta=0.01$ ) and ORCLUS (cf. Fig.11(d), parameters $k=5$ and $l=2$ reflect the cluster structure exactly) could not compete. Both missed very large and important parts of the clustering structure. This bad behavior of the two competitors can partly be explained by the high degree of noise present in the dataset. The influence of noise on the existing approaches can be observed in Fig. 12. Omitting the noise points, $4 \mathrm{C}$ is able to detect the cluster structure relatively well (cf. Fig. 12(a)) but cannot handle intersecting clusters. Even on the dataset without noise points, ORCLUS was not able to identify the five clusters correctly (cf. Fig. 12(b)). This again illustrates the superiority of CASH over existing methods especially in terms of noise robustness.

\subsection{Real-world Data}

We applied CASH on the Wages dataset ${ }^{1}$, a dataset containing average career statistics of current and former NBA players ${ }^{2}$ and a gene expression dataset [30]. The Wages data consist of $4 \mathrm{D}$ observations $(\mathrm{A}=$ age, $\mathrm{YE}=$ years of education, $\mathrm{YW}=$ years of work experience, and $\mathrm{W}$ = wage) numbering 534 from the 1985 Current Population Survey. As parameters for CASH we used $m=70$ and $s=$

\footnotetext{
${ }^{1}$ http://lib.stat.cmu.edu/datasets/CPS_85_Wages.

2 obtained from http://www.nba.com.
} 


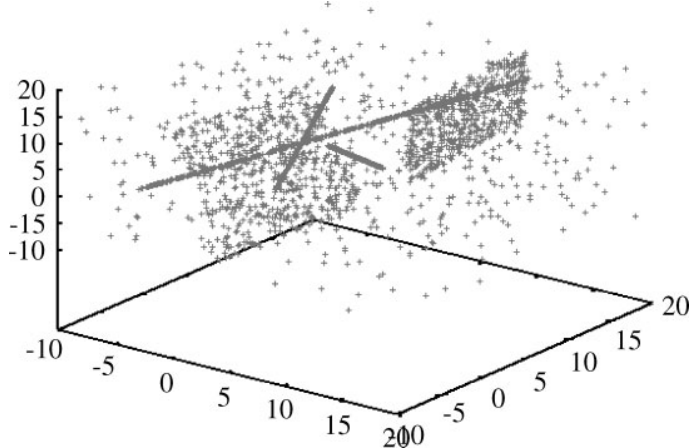

(a) Data set DS2.

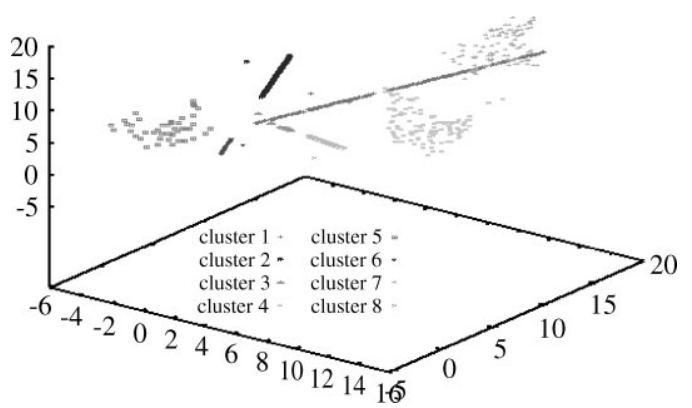

(c) 4C-Cluster 1 - 8.

Fig. 11 Clustering results on synthetic dataset DS2.

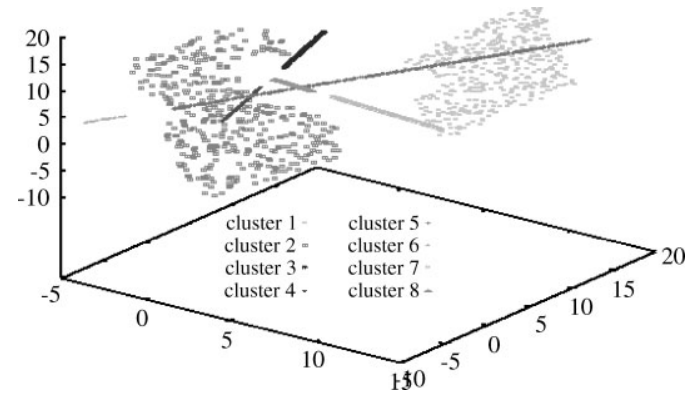

(a) $4 \mathrm{C}$ - Cluster 1 - 8 .

Fig. 12 Clustering results on DS2 after noise removal.

40. The results are summarized in Table 1: CASH detected three pure subspace clusters in this dataset, of which two data objects have been identified as noise objects. The first cluster consists only of people having 12 years of education and having started their working life at the age of 18 years. The second cluster consists only of people having 16 years of education and having started their working life at the age of 22 years. In the third cluster, only those employees are grouped, who started school at the age of 6 years, and began working immediately after graduation. Thus, the sum of years of education and work experience is equal to the age minus 6 .

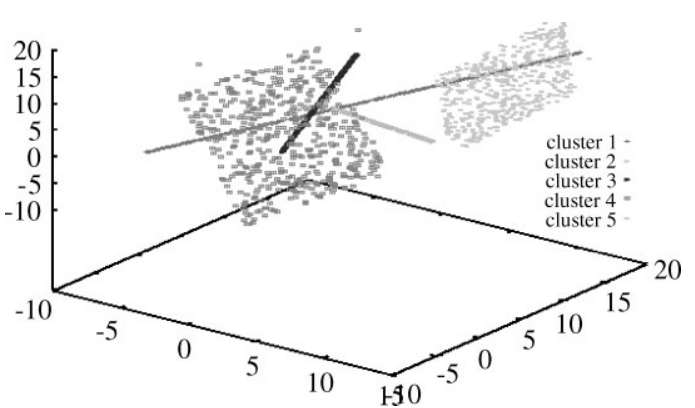

(b) CASH - Cluster 1 - 5 .

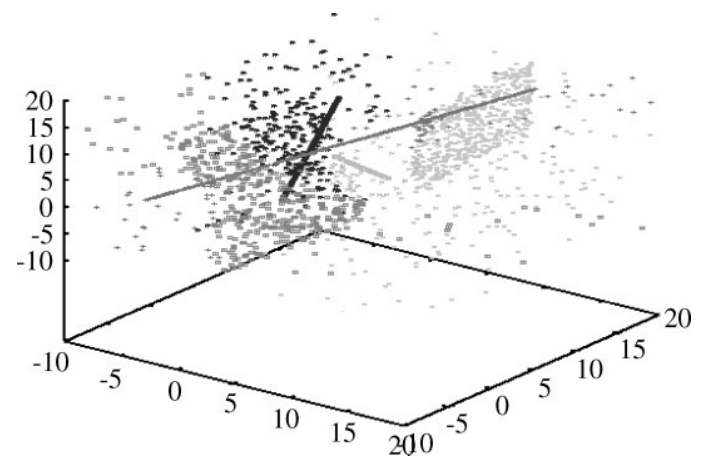

(d) ORCLUS - Cluster 1 - 5 .

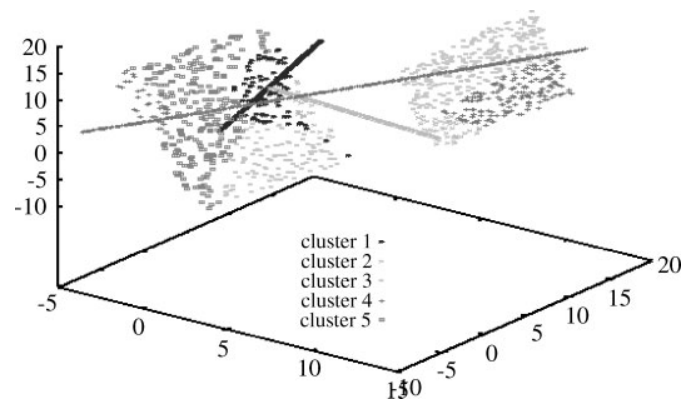

(b) ORCLUS - Cluster 1 - 5 .

Table 1. CASH clustering on wages data.

\begin{tabular}{cccc}
\hline c ID & $\operatorname{dim}$ & \# objects & Description \\
\hline 1 & 2 & 215 & YE $=12 ; \mathrm{A}-\mathrm{YW}=18$ \\
2 & 2 & 70 & $\mathrm{YE}=16 ; \mathrm{A}-\mathrm{YW}=22$ \\
3 & 3 & 247 & $\mathrm{YE}+\mathrm{YW}=\mathrm{A}-6$ \\
\hline
\end{tabular}

The NBA data contains 15 statistical measures such as 'games played' (G), 'games started' (GS), 'minutes played per game' (MPG), 'points per game' (PPG), etc. for 413 former and current NBA players. As parameters for CASH, we used $m=30$ and $s=45$. CASH detected nine interesting

Statistical Analysis and Data Mining DOI:10.1002/sam 
Table 2. CASH clustering on NBA data.

\begin{tabular}{ccl}
\hline c ID & dim & \multicolumn{1}{c}{ Description } \\
\hline 1 & 1 & 'Go-to-guys' \\
2 & 2 & Shooting guards \\
3 & 2 & Point guards \\
4 & 2 & Starting centers \\
5 & 8 & Point guards \\
6 & 9 & Power forwards \\
7 & 9 & Small forwards \\
8 & 10 & Well-known rebounder \\
9 & 12 & Role players/reserves \\
\hline
\end{tabular}

clusters of very different dimensionality each containing players of similar characteristics (cf. Table 2). In addition, several players are classified as noise. The detected correlations confirmed basketball fundamentals. For example, in cluster 1 containing superstars like Michael Jordan, Larry Bird, Shaquile O'Neal, and James Worthy, PPG of all players were negatively dependent on G and MPG. On the other hand, the more games $(\mathrm{G})$ the players were in, the higher the number of starting line-up appearances (GS). Let us note that this cluster also contains less well-known players that had similar characteristics such as Rik Smits, Dan Majerle, and Rick Fox. The three clusters containing guards all showed correlations between G and MPG on the one hand, and the number of assists and steals per game on the other hand. For the guards in cluster 3, this correlation was positive, whereas for the guards in cluster 5 , this correlation was negative. On the other hand, cluster 3 exhibits a positive correlation between the G and GS. In cluster 5 , these two attributes are also correlated but in a negative manner. This indicates that the coaches in the NBA usually decided to start with the better point guards.

In the gene expression dataset (24 dimensions, 4000 genes) CASH found several clusters of functionally related genes that are biologically interesting and relevant according to three biologically proven criteria including (i) known direct interactions of the genes or the according gene products, (ii) known common complexes of the genes or the according gene products, and (iii) participation of the according gene products in common pathways.

Neither ORCLUS nor 4C were able to detect meaningful clusters in our real-world datasets. One reason for this may be that the found clusters are highly overlapping. Thus, neither ORCLUS nor 4C can learn the appropriate similarity measure capturing the subspaces of the clusters from the local neighborhood.

\subsection{Alternative Parameterization and Cluster Hierarchies}

Last but not least, we investigated the possibilities of our novel method to produce a hierarchy of correlation clusters. We applied CASH on a three-dimensional dataset 'DS3' shown in Fig. 13(a). The dataset contains several correlation clusters including four one-dimensional clusters, a two-dimensional cluster with two embedded onedimensional clusters, and a two-dimensional cluster with one embedded one-dimensional cluster. Some noise points are also added. Not all clusters exhibit a perfect correlation, i.e. the points of the cluster deviate from the common cluster hyperplane by a small degree. In this experiment we used the alternative parameterization as described in Section 3.8 where the allowed deviation of the hyperplane can be specified independently from the allowed variance of the orientation of the cluster hyperplane. Parameters were $s=8, \delta_{-} j i t t e r=0.0011$ (specifying the allowed deviation from the cluster hyperplane), and $m=90$. With this alternative parameterization, CASH had no problems in recognizing the true cluster structure. In contrast, using the original parameterization, we could not find a parameter setting for which CASH achieved $100 \%$ accuracy. In summary, our experiments indicate that generally, the alternative parameterization achieves a similar accuracy compared to the original parameterization.

In addition, the correct relationships between all correlation clusters have been detected. The resulting hierarchy among the clusters reported by CASH is displayed in Fig.13(b). The root (level 0) of the hierarchy represents

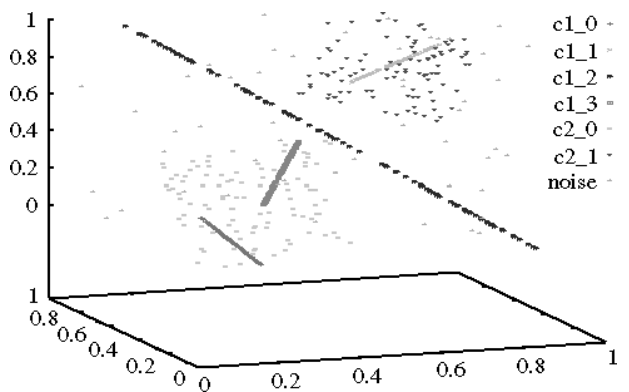

(a) Data set DS3.

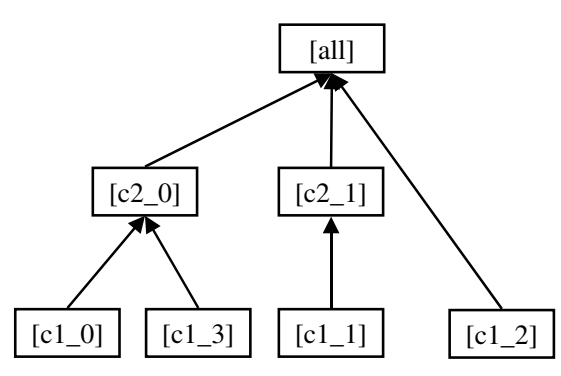

(b) Hierarchy detected by CASH.

Fig. 13 Hierarchies found on synthetic dataset DS3. 
a three-dimensional 'dummy cluster' containing the entire database denoted by 'all'. All other clusters are obviously contained in this 'cluster'. On level 1, we have the two twodimensional clusters 'c2_0' and 'c2_1'. On level 2 we have the four one-dimensional clusters. The edges indicate that clusters 'c1_0' and ' $c 1 \_3$ ' are contained in cluster 'c2_0', cluster ' $c 1_{-} 1$ ' is contained in cluster ' $c 2 \_1$ ', and cluster ' $c 1 \_2$ ' is not contained in any higher-dimensional cluster (except the root).

\section{CONCLUSIONS}

Existing subspace-clustering methods only find clusters that are dense and not noisy in the original feature space. In this article, we overcome this severe limitation by introducing $\mathrm{CASH}$, a novel approach for detecting any oriented subspace cluster regardless of its density even in a very noisy environment. CASH also features the illustration of containment-relationships among the correlation clusters, and the derivation of a quantitative model for a user-friendly explanation of the detected clusters. Our experimental evaluation confirms that $\mathrm{CASH}$ significantly outperforms competing approaches in terms of robustness and effectiveness. Although CASH can only be regarded as a first step in the direction of a global correlation clustering due to its rather high time and space complexity, it overcomes the fundamental limitation of existing approaches to correlation clustering, the locality assumption. We therefore hope to stimulate further research in the direction of global correlation clustering.

$$
\begin{aligned}
& \frac{\partial f_{p}}{\partial \alpha_{n}}(\alpha)=\prod_{i=1}^{n-1} \sin \left(\alpha_{i}\right) \\
& \cdot\left(-p_{n} \cdot \sin \left(\alpha_{n}\right)+\sum_{j=n+1}^{d} p_{j} \cdot \cos \left(\alpha_{n}\right)\right. \\
& \left.\cdot\left[\prod_{k=n+1}^{j-1} \sin \left(\alpha_{k}\right)\right] \cdot \cos \left(\alpha_{j}\right)\right)
\end{aligned}
$$

For any first-order partial derivative $\frac{\partial f p}{\partial \alpha_{n}}(\tilde{\alpha}) \doteq 0,(1 \leq n \leq d-1)$, one of the following conditions holds:

$$
\begin{aligned}
\sin \left(\tilde{\alpha}_{1}\right) & =0 \\
& \vdots \\
\sin \left(\tilde{\alpha}_{n-1}\right) & =0 \\
\tan \left(\tilde{\alpha}_{n}\right) & =\frac{\sum_{j=n+1}^{d} p_{j} \cdot\left[\prod_{k=n+1}^{j-1} \sin \left(\tilde{\alpha}_{k}\right)\right] \cdot \cos \left(\tilde{\alpha}_{j}\right)}{p_{n}}
\end{aligned}
$$

Since the first $n-1$ conditions yield an indefinite Hessian matrix, according to the last condition, a point $\tilde{\alpha}=\left(\tilde{\alpha}_{1}, \ldots, \tilde{\alpha}_{d-1}\right)$ can be an extremum point of parameterization function $f_{p}$ only if

$$
\tilde{\alpha}_{n}=\arctan \left(\frac{\sum_{j=n+1}^{d} p_{j} \cdot\left[\prod_{k=n+1}^{j-1} \sin \left(\tilde{\alpha}_{k}\right)\right] \cdot \cos \left(\tilde{\alpha}_{j}\right)}{p_{n}}\right)
$$

As noted above, $f_{p}$ is guaranteed to have exactly one global extremum $f_{p}\left(\tilde{\alpha}_{1}, \ldots, \tilde{\alpha}_{d-1}\right)$ in $[0, \pi)^{d-1}$. The values for the angles $\tilde{\alpha}_{n}, n=$ $1, \ldots, d-1$ of the global extremum are provided by the equation given above.

\section{B. MINIMUM AND MAXIMUM VALUE}

Let $\check{\alpha}=\left(\check{\alpha}_{1}, \ldots, \check{\alpha}_{d-1}\right)$ and $\hat{\alpha}=\left(\hat{\alpha}_{1}, \ldots, \hat{\alpha}_{d-1}\right)$ for a given interval $[\check{\alpha}, \hat{\alpha}) \subseteq[0, \pi)^{d-1}, 1 \leq i \leq d-1$. To determine the point $\alpha^{\text {min }}=$ $\left(\alpha_{1}^{\min }, \ldots, \alpha_{d-1}^{\min }\right)$ where the parameterization function $f_{p}$ has a minimum in interval $[\check{\alpha}, \hat{\alpha})$ the following steps for each dimension $n=d-1, \ldots, 1$ have to be performed:

1. Let

$$
\bar{\alpha}_{n}=\arctan \left(\frac{\sum_{j=n+1}^{d} p_{j} \cdot\left[\prod_{k=n+1}^{j-1} \sin \left(\alpha_{k}^{\min }\right)\right] \cdot \cos \left(\alpha_{j}^{\min }\right)}{p_{n}}\right)
$$

be the value where $f_{p}$ has an extremum on the $n$-th axis under the constraint of known minimum angles $\alpha_{n+1}^{\min }, \ldots, \alpha_{d-1}^{\min }$.

2. Given $\alpha=\left(c_{1}, \ldots, c_{n-1}, \bar{\alpha}_{n}, \alpha_{n+1}^{\min }, \ldots, \alpha_{d-1}^{\min }\right) \in[\check{\alpha}, \hat{\alpha})^{n-1} \times[0, \pi) \times$ $[\check{\alpha}, \hat{\alpha})^{d-1-n}$, where $c_{i}$ are arbitrarily chosen values in $[\check{\alpha}, \hat{\alpha})$, we differentiate the following cases:

Statistical Analysis and Data Mining DOI:10.1002/sam
Each parameterization function $f_{p}$ is a sinusoid with a period of $2 \pi$.
Thus, any $f_{p}$ has exactly one global extremum in the interval $[0, \pi)^{d-1}$. To find the global extremum of $f_{p}$, those angles $\alpha_{1}, \ldots, \alpha_{d-1}$ need to be determined where all the first-order derivatives of $f_{p}$ are zero, and the Hessian matrix is either positive or negative definite. The first-order partial derivatives of the parameterization function $f_{p}$ are given by: 


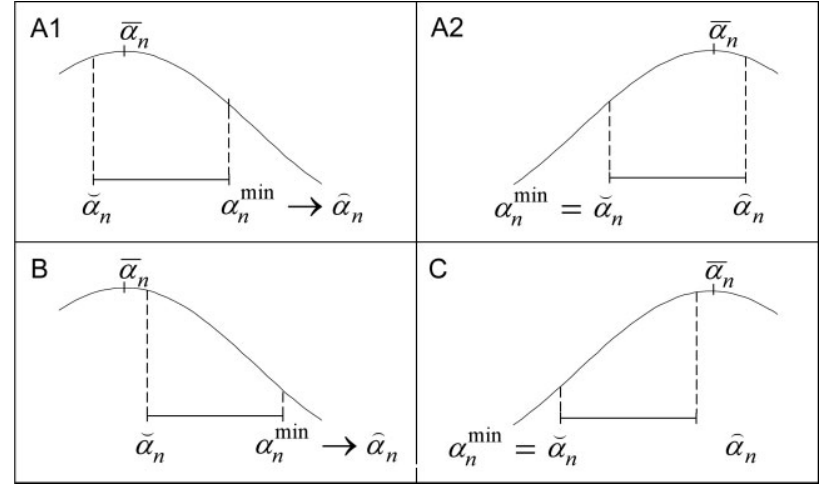

Fig. 14 Different cases for finding the minimum of a parameterization function in a given interval.

i. $f_{p}$ has a maximum in $\alpha$ :

$$
\begin{aligned}
& \text { A. } \check{\alpha}_{n} \leq \bar{\alpha}_{n} \leq \hat{\alpha}_{n}: \\
& \text { A1. } \bar{\alpha}_{n}-\check{\alpha}_{n} \leq \hat{\alpha}_{n}-\bar{\alpha}_{n}: \alpha_{n}^{\min } \rightarrow \hat{\alpha}_{n} . \\
& \text { A2. } \bar{\alpha}_{n}-\check{\alpha}_{n}>\hat{\alpha}_{n}-\bar{\alpha}_{n}: \alpha_{n}^{\min }=\check{\alpha}_{n} . \\
& \text { B. } \bar{\alpha}_{n}<\check{\alpha}_{n}: \alpha_{n}^{\min } \rightarrow \hat{\alpha}_{n} . \\
& \text { C. } \bar{\alpha}_{n}>\hat{\alpha}_{n}: \alpha_{n}^{\min }=\check{\alpha}_{n} .
\end{aligned}
$$

As illustrated in Fig. 14, if $\bar{\alpha}_{n}$ is inside the interval and nearer to the left boundary (A1), the minimum value $\alpha_{n}^{\min }$ is located at the right boundary and vice versa (A2). If $\bar{\alpha}_{n}$ is outside the interval (B and C), the minimum value $\alpha_{n}^{\min }$ is located at the opposite boundary.

ii. $f_{p}$ has a minimum in $\alpha$ : The same principle of reasoning has to be applied contrarilywise.
A. $\check{\alpha}_{n} \leq \bar{\alpha}_{n} \leq \hat{\alpha}_{n}: \alpha_{n}^{\min }=\bar{\alpha}_{n}$.
B. $\bar{\alpha}_{n}<\check{\alpha}_{n}: \alpha_{n}^{\min }=\check{\alpha}_{n}$.
C. $\bar{\alpha}_{n}>\hat{\alpha}_{n}: \alpha_{n}^{\min } \rightarrow \hat{\alpha}_{n}$.

The maximum $\alpha^{\max }=\left(\alpha_{1}^{\max }, \ldots, \alpha_{d-1}^{\max }\right)$ of $f_{p}$ in a given interval $[\check{\alpha}, \hat{\alpha}) \subseteq[0, \pi)^{d-1}$ can be determined analogously.

\section{REFERENCES}

[1] R. Agrawal, J. Gehrke, D. Gunopulos, and P. Raghavan, Automatic subspace clustering of high dimensional data for data mining applications, In Proceedings of the ACM International Conference on Management of Data (SIGMOD), Seattle, 1998.

[2] K. Kailing, H.-P. Kriegel, and P. Kröger, Densityconnected subspace clustering for high-dimensional data, In Proceedings of the 4th SIAM International Conference on Data Mining (SDM), Orlando, 2004.

[3] C. C. Aggarwal, C. M. Procopiuc, J. L. Wolf, P. S. Yu, and J. S. Park, Fast algorithms for projected clustering, In Proceedings of the ACM International Conference on Management of Data (SIGMOD), Philadelphia, 1999.
[4] C. M. Procopiuc, M. Jones, P. K. Agarwal, and T. M. Murali, A Monte Carlo algorithm for fast projective clustering, In Proceedings of the ACM International Conference on Management of Data (SIGMOD), Madison, 2002.

[5] C. Böhm, K. Kailing, H.-P. Kriegel, and P. Kröger, Density connected clustering with local subspace preferences, In Proceedings of the 4th International Conference on Data Mining (ICDM), Brighton, 2004.

[6] C. C. Aggarwal and P. S. Yu, Finding generalized projected clusters in high dimensional space, In Proceedings of the ACM International Conference on Management of Data (SIGMOD), Dallas, 2000.

[7] C. Böhm, K. Kailing, P. Kröger, and A. Zimek, Computing clusters of correlation connected objects, In Proceedings of the ACM International Conference on Management of Data (SIGMOD), Paris, 2004.

[8] A. K. H. Tung, X. Xu, and C. B. Ooi, CURLER: Finding and visualizing nonlinear correlated clusters, In Proceedings of the ACM International Conference on Management of Data (SIGMOD), Baltimore, 2005.

[9] K. Beyer, J. Goldstein, R. Ramakrishnan, and U. Shaft, When is "nearest neighbor" meaningful? In Proceedings of the 7th International Conference on Database Theory (ICDT), Jerusalem, 1999.

[10] A. Hinneburg, C. C. Aggarwal, and D. A. Keim, What is the nearest neighbor in high dimensional spaces? In Proceedings of the 26th International Conference on Very Large Data Bases (VLDB), Cairo, 2000.

[11] C. C. Aggarwal, A. Hinneburg, and D. Keim, On the surprising behavior of distance metrics in high dimensional space, In Proceedings of the 8th International Conference on Database Theory (ICDT), London, 2001.

[12] H.-P. Kriegel, P. Kröger, E. Schubert, and A. Zimek, A general framework for increasing the robustness of PCAbased correlation clustering algorithms, In Proceedings of the 20th International Conference on Scientific and Statistical Database Management (SSDBM), Hong Kong, 2008.

[13] E. Achtert, C. Böhm, H.-P. Kriegel, P. Kröger, I. MüllerGorman, and A. Zimek, Finding hierarchies of subspace clusters, In Proceedings of the 10th European Conference on Principles of Knowledge Discovery and Data Mining (PKDD), Berlin, 2006.

[14] E. Achtert, C. Böhm, H.-P. Kriegel, P. Kröger, I. MüllerGorman, and A. Zimek, Detection and visualization of subspace cluster hierarchies, In Proceedings of the 12th International Conference on Database Systems for Advanced Applications (DASFAA), Bangkok, 2007.

[15] Y. Cheng and G. M. Church, Biclustering of expression data, In Proceedings of the 8th International Conference on Intelligent Systems for Molecular Biology (ISMB), San Diego, 2000.

[16] J. Yang, W. Wang, H. Wang, and P. S. Yu, $\delta$-clusters: Capturing subspace correlation in a large data set, In Proceedings of the 18th International Conference on Data Engineering (ICDE), San Jose, 2002.

[17] H. Wang, W. Wang, J. Yang, and P. S. Yu, Clustering by pattern similarity in large data sets, In Proceedings of the ACM International Conference on Management of Data (SIGMOD), Madison, 2002.

[18] J. Pei, X. Zhang, M. Cho, H. Wang, and P. S. Yu, MaPle: A fast algorithm for maximal pattern-based clustering, In Proceedings of the 3th International Conference on Data Mining (ICDM), Melbourne, 2003.

[19] J. Liu and W. Wang, OP-Cluster: Clustering by tendency in high dimensional spaces, In Proceedings of the 
3th International Conference on Data Mining (ICDM), Melbourne, 2003.

[20] K. Chakrabarti and S. Mehrotra, Local dimensionality reduction: A new approach to indexing high dimensional spaces, In Proceedings of the 26th International Conference on Very Large Data Bases (VLDB), Cairo, 2000.

[21] E. Achtert, C. Böhm, P. Kröger, and A. Zimek, Mining hierarchies of correlation clusters, In Proceedings of the 18th International Conference on Scientific and Statistical Database Management (SSDBM), Vienna, 2006.

[22] E. Achtert, C. Böhm, H.-P. Kriegel, P. Kröger, and A. Zimek, Robust, complete, and efficient correlation clustering, In Proceedings of the 7th SIAM International Conference on Data Mining (SDM), Minneapolis, 2007.

[23] E. Achtert, C. Böhm, H.-P. Kriegel, P. Kröger, and A. Zimek, On exploring complex relationships of correlation clusters, In Proceedings of the 19th International Conference on Scientific and Statistical Database Management (SSDBM), Banff, 2007.

[24] N. Bansal, A. Blum, and S. Chawla, Correlation clustering, Mach Learn 56 (2004), 89-113.
[25] P. V. C. Hough, Methods and means for recognizing complex patterns, U.S. Patent 3069654, December 18 1962.

[26] A. Rosenfeld, Picture Processing by Computer, CSUR 1(3) (1969), 147-176.

[27] R. O. Duda and P. E. Hart, Use of the Hough transformation to detect lines and curves in pictures, Commun ACM 15(1) (1972), 11-15.

[28] K. S. Miller, Multidimensional Gaussian Distributions, John Wiley and Sons, New York, 1964.

[29] E. Achtert, C. Böhm, H.-P. Kriegel, P. Kröger, and A. Zimek, Deriving quantitative models for correlation clusters, In Proceedings of the 12th ACM International Conference on Knowledge Discovery and Data Mining (SIGKDD), Philadelphia, 2006.

[30] P. T. Spellman, G. Sherlock, M. Q. Zhang, V. R. Iyer, K. Anders, M. B. Eisen, P. O. Brown, D. Botstein, and B. Futcher, Comprehensive identification of cell cycleregulated genes of the yeast saccharomyces cerevisiae by microarray hybridization, Mol Biol Cell 9 (1998), 3273-3297. 